\title{
Connection between the renormalization groups of Stückelberg-Petermann and Wilson
}

\author{
Michael Dütsch ${ }^{1,2}$ \\ August 21, 2018 \\ ${ }^{1}$ Institut für Theoretische Physik, Universität Göttingen, \\ Friedrich-Hund-Platz 1, 37077 Göttingen, Germany \\ ${ }^{2}$ Courant Research Centre "Higher Order Structures in Mathematics", \\ Universität Göttingen, Bunsenstr. 3-5, 37073 Göttingen, Germany \\ michael.duetsch@theorie.physik.uni-goe.de
}

\begin{abstract}
The Stückelberg-Petermann renormalization group is the group of finite renormalizations of the $S$-matrix in the framework of causal perturbation theory. The renormalization group in the sense of Wilson relies usually on a functional integral formalism, it describes the dependence of the theory on a UV-cutoff $\Lambda$; a widespread procedure is to construct the theory by solving Polchinski's flow equation for the effective potential.

To clarify the connection between these different approaches we proceed as follows: in the framework of causal perturbation theory we introduce an UV-cutoff $\Lambda$, define an effective potential $V_{\Lambda}$, prove a pertinent flow equation and compare with the corresponding terms in the functional integral formalism. The flow of $V_{\Lambda}$ is a version of Wilson's renormalization group. The restriction of these operators to local interactions can be approximated by a subfamily of the StückelbergPetermann renormalization group.
\end{abstract}




\section{Introduction}

There are different versions of the renormalization group (RG), however their relations are not completely understood. The aim of this article is to clarify the connection between the renormalization groups of StückelbergPetermann and Wilson in the framework of perturbation theory. In order that this paper is better intelligible for physicists we use sometimes a graphical language and omit some mathematical technicalities, for a more mathematical formulation we refer to [2].

By the Stückelberg-Petermann RG and Wilson's RG we mean the following.

- The Stückelberg - Petermann $R G \mathcal{R}[16]$ is the version of the $\mathrm{RG}$ as it appears in causal perturbation theory $[13,9,6,7,1,2]$. It relies on the non-uniqueness of the $S$-matrix $\mathbf{S}$. Roughly speaking the Main Theorem states that a change $\mathbf{S} \rightarrow \hat{\mathbf{S}}$ of the renormalization presription can be absorbed in a renormalization of the interaction $V \rightarrow Z(V)$ [13]:

$$
\hat{\mathbf{S}}(V)=\mathbf{S}(Z(V)) \quad \forall V \in \mathcal{F}_{\text {loc }}
$$

(where $\mathcal{F}_{\text {loc }}$ is the space of all local interactions). The Stückelberg - Petermann RG $\mathcal{R}$ is the set of all bijective maps $Z: \mathcal{F}_{\text {loc }} \rightarrow \mathcal{F}_{\text {loc }}$ appearing in this relation when $\mathbf{S}$ and $\hat{\mathbf{S}}$ run through all admissible $S$-matrices. From the complete statement of the Main Theorem $[6,7]$ (see Sect. 3.2) it follows that $\mathcal{R}$ is indeed a group; due to (1.1) $\mathcal{R}$ can be interpreted as the group of finite renormalizations of the $S$-matrix.

- The $R G$ in the sense of Wilson relies usually on a functional integral approach, it describes the dependence of the theory on a cutoff $\Lambda$, which one introduces to avoid UV-divergences. We imitate this approach in the framework of causal perturbation theory by proceeding as follows [2]: Let $p_{\Lambda}$ be a regularized Feynman propagator, which converges (in an appropriate sense) to the Feynman propagator for $\Lambda \rightarrow \infty$. In terms of $p_{\Lambda}$ we construct a regularized $S$-matrix $\mathbf{S}_{\Lambda}$. With that we define the effective potential $V_{\Lambda}$ at scale $\Lambda$ as a function of the original interaction $V$ by the condition that the cutoff theory with interaction $V_{\Lambda}$ agrees with the exact theory with interaction $\mathrm{V}$, that is

$$
\mathbf{S}_{\Lambda}\left(V_{\Lambda}\right)=\mathbf{S}(V) \quad \text { or explicitly } \quad V_{\Lambda}:=\mathbf{S}_{\Lambda}^{-1} \circ \mathbf{S}(V) .
$$


Here we use that $\mathbf{S}_{\Lambda}$ is invertible (see [2] and Sect. 4). We point out that in general $V_{\Lambda}$ is a non-local interaction.

The problem with this definition is that usually $\mathbf{S}$ is unknown. Therefore, one computes $V_{\Lambda}$ by solving Polchinski's flow equation [12, 14, 11], which can be derived from the definition (1.2) (see Sect. 5 and [2]) and has the form

$$
\frac{d}{d \Lambda} V_{\Lambda}=F_{\Lambda}\left(V_{\Lambda} \otimes V_{\Lambda}\right),
$$

where $F_{\Lambda}$ is linear and explicitly known. Integration of the flow equation yields $V_{\Lambda}$ and from that the $S$-matrix is obtained by $\mathbf{S}(V)=$ $\mathbf{S}_{\Lambda}\left(V_{\Lambda}\right)$; in practice it is easier to compute

$$
\lim _{\Lambda \rightarrow \infty} \mathbf{S}_{\Lambda}\left(V_{\Lambda}\right),
$$

since several terms vanish in this limit and, hence, need not to be computed in detail.

We point out that there is no group structure in the mathematical sense in Wilson's RG.

\section{Star-product quantization}

We use a formalism which arises when applying deformation quantization to the underlying free classical fields $[4,5]$.

To simplify the notations we restrict this paper to a real scalar field $\varphi$ in $d$-dimensional Minkowski space. We work with an "off-shell formalism" which means that the classical field configuration space is $C^{\infty}\left(\mathbb{R}^{d}\right)$ (and not only the space of solutions of the field equations).

We define the space $\mathcal{F}$ of observables as the set of all functionals $F$ : $C^{\infty}\left(\mathbb{R}^{d}\right) \rightarrow \mathbb{C}$, which are infinitely differentiable and all functional derivatives $\frac{\delta^{n} F}{\delta \varphi^{n}}(n \in \mathbb{N})$ must be distributions with compact supports. There is an additional defining condition on the wave front sets $\operatorname{WF}\left(\frac{\delta^{n} F}{\delta \varphi^{n}}\right), n \in \mathbb{N}$, which is a microlocal version of translation invariance $[4,5,6,2]$.

The following subspaces of $\mathcal{F}$ will be of crucial importance.

- The non-local functionals $\mathcal{F}_{0}$ are defined by the stronger requirement that $\frac{\delta^{n} F}{\delta \varphi^{n}}$ is a smooth function with compact support for all $n \in \mathbb{N}$. 
- The local functionals $\mathcal{F}_{\text {loc }}$ are defined by the additional condition that $\frac{\delta^{n} F}{\delta \varphi^{n}}\left(x_{1}, \ldots, x_{n}\right)=0$ if $x_{i} \neq x_{j}$ for some $(i, j)$.

Local interactions are usually of the form

$$
F(\varphi)=\int d x f(x) L(\varphi(x), \partial \varphi(x), \partial \partial \varphi(x), \ldots) \in \mathcal{F}_{\text {loc }}
$$

where $f \in \mathcal{D}\left(\mathbb{R}^{d}\right)$ switches the interaction and $L \in \mathcal{C}^{\infty}$ does not need to be a polynomial.

By the support of an observable $F$ we mean $\operatorname{supp} F:=\operatorname{supp} \frac{\delta F}{\delta \varphi}$.

The pointwise product

$$
(F \cdot G)(\varphi):=F(\varphi) \cdot G(\varphi)
$$

is commutative, we call it also the classical product.

To obtain the Poisson algebra of free fields we define the Poisson bracket. For this purpose we need the retarded propagator $\Delta_{R}$ of the KleinGordon operator, from which we construct the commutator function

$$
\Delta(x):=\Delta_{R}(x)-\Delta_{R}(-x)=-\Delta(-x) .
$$

Graphically the Poisson bracket of $F, G \in \mathcal{F}$ is defined by contracting once $F$ with $G$ with propagator $\Delta$, i.e.

$$
\{F, G\}:=\int d x d y \frac{\delta F}{\delta \varphi(x)} \Delta(x-y) \frac{\delta G}{\delta \varphi(y)} .
$$

In view of a $\star$-product quantization we introduce $\mathcal{F}[[\hbar]]$ as the space of formal power series in $\hbar$ with coefficients in $\mathcal{F}$ (and similar for subspaces of $\mathcal{F})$.

Now we define the 'product with propagator $p$ '

$$
\star_{p}: \mathcal{F}_{1}[[\hbar]] \times \mathcal{F}_{1}[[\hbar]] \rightarrow \mathcal{F}[[\hbar]] ;(F, G) \mapsto F \star_{p} G
$$

(where $\mathcal{F}_{1}$ is a subspace of $\mathcal{F}$ and $p \in \mathcal{S}^{\prime}\left(\mathbb{R}^{d}\right)$ is a distribution with suitable properties, see the examples below) by the prescription

$$
\begin{aligned}
F \star_{p} G:= & \sum_{n \geq 0} \frac{\hbar^{n}}{n !} \int d x_{1} \ldots d y_{1} \ldots \frac{\delta^{n} F}{\delta \varphi\left(x_{1}\right) \ldots \delta \varphi\left(x_{n}\right)} \\
& p\left(x_{1}-y_{1}\right) \ldots p\left(x_{n}-y_{n}\right) \frac{\delta^{n} G}{\delta \varphi\left(y_{1}\right) \ldots \delta \varphi\left(y_{n}\right)} .
\end{aligned}
$$


The zeroth term $(n=0)$ is the classical product. The $n$-th term has prercisely $n$ contractions of $F$ with $G$, each contraction with the propagator $p$. Hence, $F \star_{p} G$ is the sum over all possible contractions of $F$ with $G$. For non-local functionals $\left(\mathcal{F}_{1}=\mathcal{F}_{0}\right)$ the integral in $(2.6)$ exists for all $p \in \mathcal{S}^{\prime}\left(\mathbb{R}^{d}\right)$ (it means smearing of the distribution $p \otimes \ldots \otimes p$ with the test function $\left.\frac{\delta^{n} F}{\delta \varphi\left(x_{1}\right) \ldots} \cdot \frac{\delta^{n} G}{\delta \varphi\left(y_{1}\right) \ldots}\right)$. One can show that $\star_{p}$ is associative, obviously it is distributive.

The following examples for $\star_{p}$ are of crucial importance.

- $\star$-product quantization. We choose for $p$ a Hadamard function $H \equiv$ $H_{m}$. A Hadamard function is a Poincaré invariant solution of the Klein Gordon equation with mass $m$, which is smooth in $m \geq 0$ and fulfils

(i) $H(z)-H(-z)=i \Delta(z)$ and

(ii) $z \mapsto\left(H(z)-\Delta_{+}(z)\right) \quad$ is smooth, where $\Delta_{+}$is the Wightman 2point function. Since powers $\left(\Delta_{+}(z)\right)^{n}$ exist, this condition implies that $(H(z))^{n}$ exists $\forall n \in \mathbb{N}$.

$F \star_{H} G$ exists also for local functionals, i.e. it exists $\forall F, G \in \mathcal{F}$. Namely, property (ii) and the wave front set property of $\frac{\delta^{n} F}{\delta \varphi^{n}}$ and $\frac{\delta^{n} G}{\delta \varphi^{n}}$ imply that the appearing products of distributions exist [4].

The product $\star_{H}$ viewed as a map $\mathcal{F} \times \mathcal{F} \rightarrow \mathcal{F}[[\hbar]]$ (i.e. the arguments are $\sim \hbar^{0}$ ) is a $\star$-product, i.e. $F \star_{H} G$ is a $\hbar$-dependent deformation of $F \cdot G$,

$$
\lim _{\hbar \rightarrow 0} F \star_{H} G=F \cdot G
$$

with

$$
\lim _{\hbar \rightarrow 0} \frac{1}{i \hbar}\left(F \star_{H} G-G \star_{H} F\right)=\{F, G\} .
$$

The validity of the last relation relies on property (i) of $H$. The Wightman 2-point function $\Delta_{+}$yields also a $\star$-product $\star_{\Delta_{+}}$, but $\Delta_{+}$is not smooth in $m$ at $m=0$.

- Time ordered product of nonlocal functionals. The time ordered product with respect to the $\star$-product $\star_{H}$ must satisfy

$$
T(\varphi(x) \varphi(y)):=\left\{\begin{array}{lll}
\varphi(x) \star_{H} \varphi(y) & \text { if } & x^{0}>y^{0} \\
\varphi(y) \star_{H} \varphi(x) & \text { if } & y^{0}>x^{0}
\end{array}\right.
$$


These two cases can be summarized as

$$
T(\varphi(x) \varphi(y))=\varphi(x) \star_{H_{F}} \varphi(y)
$$

with

$$
H_{F}(z):=\Theta\left(z^{0}\right) H(z)+\Theta\left(-z^{0}\right) H(-z)\left(=H_{F}(-z)\right) .
$$

Hence, the propagator for the time ordered product w.r.t. $\star_{H}$ is $H_{F}$ and, therefore, we define the time ordered product of non-local functionals as the product with propagator $H_{F}$ :

$$
T\left(F_{1} \otimes \ldots \otimes F_{n}\right):=F_{1} \star_{H_{F}} \ldots \star_{H_{F}} F_{n}, \quad \forall F_{1}, \ldots, F_{n} \in \mathcal{F}_{0} .
$$

Since $H_{F}(-z)=H_{F}(z)$, this time ordered product is commutative, hence it cannot be a $\star$-product (due to (2.8)).

\section{The Stückelberg - Petermann renormalization group}

\subsection{Time ordered product of local functionals}

For simplicity we assume from now on that all observables $F \in \mathcal{F}$ are polynomial in $\varphi$ and its derivatives, and we write $\mathcal{F}$ for $\mathcal{F}[[\hbar]]$ (and similar for subspaces of $\mathcal{F}$ ).

Trying to extend the definition (2.12) to local functionals, powers $\left(H_{F}(z)\right)^{n}$ and terms like $H_{F}\left(z_{1}\right) H_{F}\left(z_{2}\right) H_{F}\left(z_{1}+z_{2}\right)$ appear, which do not exist in $d \geq 4$ or $d \geq 6$ dimensions! (These are the famous UV-divergences of perturbative QFT.) Therefore, we define the time ordered product of local functionals in an alternative, axiomatic way: we require that the time ordered product of $n$-th order

$$
T_{n}: \mathcal{F}_{\text {loc }}^{\otimes n} \rightarrow \mathcal{F}
$$

is a linear and totally symmetric map. With that the defining axioms can be given in terms of the generating functional - the $S$-matrix

$$
\mathbf{S}: \mathcal{F}_{\text {loc }} \rightarrow \mathcal{F} ; \mathbf{S}(V):=\sum_{n=0}^{\infty} \frac{T_{n}\left(V^{\otimes n}\right)}{n !} .
$$

Or vice versa $T_{n}$ is obtained from $\mathbf{S}$ by

$$
T_{n}\left(V^{\otimes n}\right)=\left.\mathbf{S}^{(n)}(0)\left(V^{\otimes n}\right) \equiv \frac{d^{n}}{d \lambda^{n}} \mathbf{S}(\lambda V)\right|_{\lambda=0}
$$


(where $\mathbf{S}^{(n)}(0)$ denotes the $n$-th derivative of $\mathbf{S}$ at the origin). We use the axioms of causal perturbation theory $[8,15,3]^{1}$

Causality: $\mathbf{S}(A+B)=\mathbf{S}(A) \star_{H_{m}} \mathbf{S}(B)$ if $\operatorname{supp} A$ is later than $\operatorname{supp} B$, i.e. supp $A \cap\left(\operatorname{supp} B+\bar{V}_{-}\right)=\emptyset$ (where $\bar{V}_{-}$denotes the full and closed backward light cone).

Starting element: $\mathbf{S}(0)=1, \mathbf{S}^{(1)}(0)=\mathrm{id}$.

Field Independence: $\delta \mathbf{S} / \delta \varphi=0$.

\section{Poincaré invariance}

Unitarity: $\overline{\mathbf{S}(-V)} \star_{H_{m}} \mathbf{S}(\bar{V})=1$ (where the bar means complex conjugation).

Smoothness in $m: \mathbf{S}$ depends smoothly on the mass $m$ of the free theory $\forall m \geq 0$.

Scaling: $\mathbf{S}$ scales almost homogeneously under $(x, m) \mapsto\left(\rho x, \rho^{-1} m\right)$, by which we mean that homogeneous scaling (holding for the corresponding classical theory) is maintained up to powers of $\log \rho$.

Note that in the Causality condition the $\star$-product w.r.t. a Hadamard function $H_{m}$ appears. It is here where the information about the free field equation (in particular about the value of the mass $m \geq 0$ ) enters the axioms. If $H$ would be replaced by $\Delta_{+}$, Smoothness in $m$ would be violated at $m=0$.

Epstein and Glaser showed that these axioms have a solution [8] (for somewhat alternative proofs see $[15,3,6])$ : they gave a construction of the time ordered products $T_{n}$ by induction on $n$. In this construction renormalization appears as the problem of extending the distributional kernels of $T_{n}$ from $\mathcal{D}\left(\mathbb{R}^{d n} \backslash \Delta_{n}\right)$ to $\mathcal{D}\left(\mathbb{R}^{d n}\right)$ where $\Delta_{n}:=\left\{\left(x_{1}, \ldots, x_{n}\right) \in \mathbb{R}^{d n} \mid x_{1}=x_{2}=\ldots=x_{n}\right\}$. The non-uniqueness of this extension is the reason for the non-uniqueness of the $S$-matrix.

'Causality' and 'Starting element' are the basic axioms; the other axioms are not mandatory, they are called (re)normalization conditions because their only purpose is to restrict the set of admissible extensions.

\footnotetext{
${ }^{1}$ In view of the generalization to curved spacetimes we work with a somewhat modified version of the axioms given in [6].
} 


\subsection{Non-uniqueness of the $S$-matrix}

We define the Stückelberg - Petermann RG $\mathcal{R}$ as the set of all analytic bijections $Z: \mathcal{F}_{\text {loc }} \rightarrow \mathcal{F}_{\text {loc }}$ with

Starting element: $Z(0)=0, \quad Z^{(1)}(0)=\mathrm{id}, \quad Z=\mathrm{id}+O(\hbar)$.

Locality: $Z$ is local in the sense that

$Z(A+B+C)=Z(A+B)-Z(B)+Z(B+C)$

if $\operatorname{supp} A \cap \operatorname{supp} C=\emptyset$.

Field Independence: $\delta Z / \delta \varphi=0$.

Poincaré invariance

Unitarity: $\bar{Z}(-V)+Z(V)=0$.

Smoothness in $m: Z$ depends smoothly on $m \geq 0$.

Scaling: $Z$ scales almost homogeneously under $(x, m) \mapsto\left(\rho x, \rho^{-1} m\right)$.

Every renormalization condition on $\mathbf{S}$ has a corresponding requirement on $Z$. Analyticity of $Z$ means that it is given by its Taylor series:

$$
Z(V)=\sum_{n=1}^{\infty} \frac{Z^{(n)}(0)\left(V^{\otimes n}\right)}{n !}
$$

The 'Main Theorem' describes the non-uniqueness of the $S$-matrix in terms of the Stückelberg - Petermann RG $\mathcal{R}$.

Theorem 1. (i) Given two renormalization prescriptions $\mathbf{S}$ and $\hat{\mathbf{S}}$ there exists a unique map $Z: \mathcal{F}_{\text {loc }} \rightarrow \mathcal{F}_{\text {loc }}$ with $Z(0)=0$ and

$$
\hat{\mathbf{S}}=\mathbf{S} \circ Z \text {. }
$$

This $Z$ is an element of the Stückelberg - Petermann $R G \mathcal{R}$.

(ii) Conversely, given an $S$-matrix $\mathbf{S}$ and an arbitrary $Z \in \mathcal{R}$, then $\hat{\mathbf{S}}:=\mathbf{S} \circ Z$ satisfies also the axioms for an $S$-matrix.

For the proof we refer to [6].

A Corollary of this Theorem states that, for $Z_{1}, Z_{2} \in \mathcal{R}$, the composition $Z_{1} \circ Z_{2}$ is also an element of $\mathcal{R}$, i.e. that $\mathcal{R}$ is indeed a group [6]. Namely, given $Z_{1}, Z_{2} \in \mathcal{R}$ and choosing an arbitrary $S$-matrix $\mathbf{S}$, part (ii) implies that $\mathbf{S}_{1}:=\mathbf{S} \circ Z_{1}$ and $\mathbf{S}_{2}:=\mathbf{S}_{1} \circ Z_{2}$ satisfy also the axioms. From $\mathbf{S}_{2}=\mathbf{S} \circ\left(Z_{1} \circ Z_{2}\right)$ and part (i) it follows that $Z_{1} \circ Z_{2} \in \mathcal{R}$. 


\section{Regularized time-ordered product}

The definition (2.12) of the time ordered product of non-local functionals can be extended to local functionals, if one regularizes the Feynman propagator $H_{F}$ by introducing a cutoff $\Lambda$.

Let $\left(p_{\Lambda}\right)_{\Lambda>0}$ be a family of test functions $\left(p_{\Lambda} \in \mathcal{S}\left(\mathbb{R}^{d}\right)\right)$ which approximates $H_{F}$, more precisely

$$
\lim _{\Lambda \rightarrow \infty} p_{\Lambda}=H_{F} \quad \text { in the Hörmander topology [10], }
$$

and for $\Lambda \rightarrow 0$ it is required that

$$
p_{0}=0 \quad \text { or } \quad \lim _{\Lambda \rightarrow 0} p_{\Lambda}=0 \quad \text { in the Hörmander topology. }
$$

With regard to $H_{F}(-z)=H_{F}(z)$, we additionally require $p_{\Lambda}(-z)=p_{\Lambda}(z)$.

The regularized time-ordered product,

$$
T_{\Lambda}\left(F^{\otimes n}\right):=F \star_{p_{\Lambda}} \cdots \star_{p_{\Lambda}} F
$$

is well-defined $\forall F \in \mathcal{F}$ since $p_{\Lambda} \in \mathcal{S}\left(\mathbb{R}^{d}\right)$.

The corresponding generating functional is the regularized $S$-matrix

$$
\mathbf{S}_{\Lambda}: \mathcal{F} \rightarrow \mathcal{F} ; \mathbf{S}_{\Lambda}(F):=\sum_{n=0}^{\infty} \frac{1}{n !} T_{\Lambda}\left(F^{\otimes n}\right)=: e_{\star_{p_{\Lambda}}}^{F}
$$

(The last expression is a suggestive short-hand notation for the series.) In contrast to the exact $S$-matrix $\mathbf{S}$, the domain of $\mathbf{S}_{\Lambda}$ is $\mathcal{F}$ (and not only $\mathcal{F}_{\text {loc }}$ ) and $\mathbf{S}_{\Lambda}$ is invertible. We also point out that $\lim _{\Lambda \rightarrow \infty} \mathbf{S}_{\Lambda}$ does not exist in general.

Proof of invertability: following [2] we write the product $\star_{p_{\Lambda}}$ alternatively as

$$
F \star_{p_{\Lambda}} G=\tau_{\Lambda}\left(\tau_{\Lambda}^{-1} F \cdot \tau_{\Lambda}^{-1} G\right)
$$

where

$$
\tau_{\Lambda} F:=\exp \left(i \hbar \Gamma_{\Lambda}\right) F
$$

with

$$
\Gamma_{\Lambda}:=\frac{1}{2} \int d x d y p_{\Lambda}(x-y) \frac{\delta^{2}}{\delta \varphi(x) \delta \varphi(y)} .
$$

Graphically $\left(\tau_{\Lambda} F\right)(\varphi)$ is the sum over all possible contractions (with propagator $p_{\Lambda}$ ) of $\varphi$ in $F(\varphi)$. Obviously, the inverse operator $\tau_{\Lambda}^{-1}$ is $\tau_{\Lambda}^{-1}=$ 
$\exp \left(-i \hbar \Gamma_{\Lambda}\right)$. Note that the operators $\tau_{\Lambda}^{-1}$ in (4.3) are needed to remove the tadepole diagrams.

With (4.3) $\mathbf{S}_{\Lambda}$ can be written as

$$
\mathbf{S}_{\Lambda}=\tau_{\Lambda} \circ \exp \circ \tau_{\Lambda}^{-1}
$$

(where $\exp F=1+F+F \cdot F / 2 !+\ldots$ ) from which it is obvious that $\mathbf{S}_{\Lambda}$ is invertible:

$$
\mathbf{S}_{\Lambda}^{-1}=\tau_{\Lambda} \circ \log \circ \tau_{\Lambda}^{-1}
$$

Examples for regularized (Feynman) propagators:

- Euklidean theory with mass $m>0$ (following [14]).

Let $K \in \mathcal{C}^{\infty}\left(\mathbb{R}_{0}^{+},[0,1]\right)$ with

$$
K(x)=\left\{\begin{array}{lll}
0 & \text { if } & x \geq 4 \\
1 & \text { if } \quad x \leq 1
\end{array}\right.
$$

and $K^{\prime}(x)<0 \forall x \in(1,4)$, i.e. $K$ is a smooth version of a step function. The Eulklidean propagator is regularized by cutting off the momenta above a scale $\Lambda$ :

$$
\hat{p}_{\Lambda}(k):=\frac{1}{(2 \pi)^{2}\left(k^{2}+m^{2}\right)} K\left(\frac{k^{2}}{\Lambda^{2}}\right) \in \mathcal{S}\left(\mathbb{R}^{d}\right) \quad\left(k^{2} \equiv k_{0}^{2}+\vec{k}^{2}\right) .
$$

It follows $p_{\Lambda}(x) \in \mathcal{S}\left(\mathbb{R}^{d}\right), p_{\Lambda}(-x)=p_{\Lambda}(x)$ and that

$$
\lim _{\Lambda \rightarrow \infty} \hat{p}_{\Lambda}(k)=\frac{1}{(2 \pi)^{2}\left(k^{2}+m^{2}\right)} \quad(=\text { Eukl. prop. }), \quad \lim _{\Lambda \rightarrow 0} \hat{p}_{\Lambda}(k)=0
$$

w.r.t. the weak topology of $\mathcal{S}^{\prime}\left(\mathbb{R}^{d}\right)$. Due to the continuity of the inverse Fourier transformation from $\mathcal{S}^{\prime}\left(\mathbb{R}^{d}\right)$ to $\mathcal{S}^{\prime}\left(\mathbb{R}^{d}\right)$, these convergence statements hold also in $x$-space w.r.t. the weak topology. They are valid also w.r.t. the Hörmander topology in $x$-space. For the Euklidean propagator $p(x):=\lim _{\Lambda \rightarrow \infty} p_{\Lambda}(x)$ one obtains

$$
p(x)=\frac{2 \pi^{\frac{d+1}{2}}}{\Gamma\left(\frac{d-1}{2}\right)} \int_{m}^{\infty} d q\left(q^{2}-m^{2}\right)^{\frac{d-3}{2}} e^{-q|x|} \in \mathcal{S}^{\prime}\left(\mathbb{R}^{d}\right),
$$

where $|x| \equiv \sqrt{x_{0}^{2}+\vec{x}^{2}}$. In low dimensions the remaining integral gives

$$
d=2: \quad p(x)=2 \pi K_{0}(m|x|)
$$


(where $K_{0}$ is a modified Bessel function of second kind) and

$$
d=3: \quad p(x)=2 \pi^{2} \frac{e^{-m|x|}}{|x|} .
$$

Both expressions have an integrable singularity at $x=0$ and decay exponentially for $|x| \rightarrow \infty$.

- $\epsilon$-regularized relativistic theory with $m>0$ (following [11]).

$\epsilon$-regularization of the relativistic theory means that the Minkowski metric is replaced by $i \eta_{\epsilon}$, where

$$
k \eta_{\epsilon} k:=k_{0}^{2}(\epsilon-i)+\vec{k}^{2}(\epsilon+i) \text { with } \epsilon>0 .
$$

Note that $k \eta_{\epsilon} k \neq 0$ for $k \neq 0$ and that

$$
\operatorname{Re}\left(k \eta_{\epsilon} k+(\epsilon+i) m^{2}\right)=\epsilon\left(k_{0}^{2}+\vec{k}^{2}+m^{2}\right) \geq \epsilon m^{2} \quad \forall k .
$$

For the Feynman propagator of the $\epsilon$-regularized relativistic theory,

$$
\hat{p}_{\epsilon}(k)=\frac{i}{(2 \pi)^{2}\left(k \eta_{\epsilon} k+(\epsilon+i) m^{2}\right)}
$$

an UV-cutoff $\Lambda$ is introduced by an exponential damping:

$$
\hat{p}_{\epsilon, \Lambda}(k):=e^{-\Lambda^{-1}\left(k \eta_{\epsilon} k+(\epsilon+i) m^{2}\right)} \hat{p}_{\epsilon}(k)=\frac{i}{(2 \pi)^{2}} \int_{\Lambda^{-1}}^{\infty} d \alpha e^{-\alpha\left(k \eta_{\epsilon} k+(\epsilon+i) m^{2}\right)} .
$$

Obviously it holds $\hat{p}_{\epsilon, \Lambda}(k) \in \mathcal{S}\left(\mathbb{R}^{d}\right)$ and hence $p_{\epsilon, \Lambda}(x) \in \mathcal{S}\left(\mathbb{R}^{d}\right)$. We also see that $p_{\epsilon, \Lambda}(-x)=p_{\epsilon, \Lambda}(x)$.

For fixed $\epsilon>0$ we find

$$
\lim _{\Lambda \rightarrow \infty} p_{\epsilon, \Lambda}(x)=p_{\epsilon}(x) \quad \text { and } \quad \lim _{\Lambda \rightarrow 0} p_{\epsilon, \Lambda}(x)=0
$$

w.r.t. the weak topology of $\mathcal{S}^{\prime}\left(\mathbb{R}^{d}\right)$ and also w.r.t. the Hörmander topology. Namely, for $\Lambda \rightarrow \infty$ the convergence behaviour is essentially similar to the Euklidean case treated above (due to (4.10)) and for $\Lambda \rightarrow 0$ the behaviour of $p_{\epsilon, \Lambda}(x)$ is dominated by a prefactor $e^{-\Lambda^{-1} \epsilon m^{2}}$.

For $\epsilon \downarrow 0$ the family $\left(\hat{p}_{\epsilon}(k)\right)_{\epsilon>0}$ of analytic functions converges to the distribution

$$
\lim _{\epsilon \downarrow 0} \hat{p}_{\epsilon}(k)=\frac{1}{(2 \pi)^{2}\left(m^{2}-k^{2}-i 0\right)} \quad(=\text { Feynman propagator }),
$$


w.r.t. the weak topology of $\mathcal{S}^{\prime}\left(\mathbb{R}^{d}\right)$ and, hence, this holds also in $x$ space: $\lim _{\epsilon \downarrow 0} p_{\epsilon}(x)=$ (Feynman propagator) in $\mathcal{S}^{\prime}\left(\mathbb{R}^{d}\right)$. Whether this holds also w.r.t. the Hörmander topology is a more difficult question, which cannot be answered with the mathematical tools explained in this paper.

In both examples the propagator

$$
p_{\Lambda, \Lambda_{0}}:=p_{\Lambda_{0}}-p_{\Lambda} \quad\left(0<\Lambda \leq \Lambda_{0}<\infty\right)
$$

has an UV-cutoff $\left(\Lambda_{0} \rightarrow \infty\right)$ and an IR-cutoff $(\Lambda \rightarrow 0)$.

\section{Effective potential and flow equation}

To define the effective potential $V_{\Lambda}$ we recall that $\mathbf{S}_{\Lambda}$ is explicitly known and invertible and that $\mathbf{S}$ exits (although it is usually unknown). With that the effective potential $V_{\Lambda}$ at scale $\Lambda$ can be defined as explained in the introduction:

$$
V_{\Lambda}:=\mathbf{S}_{\Lambda}^{-1} \circ \mathbf{S}(V) .
$$

We also recall that in general $V_{\Lambda} \notin \mathcal{F}_{\text {loc }}$.

Similarly to $\mathbf{S}(V), V_{\Lambda}$ can be viewed as a formal power series in $\hbar$, or in $V$, or in both. For the lowest terms of the expansion in $V$ we obtain

$$
V_{\Lambda}=V+\mathcal{O}\left(V^{2}\right)
$$

by using the axiom Starting element and (4.7).

In particular for $\Lambda=0$ we obtain

$$
V_{0}=\log \circ \mathbf{S}(V)
$$

(due to $\mathbf{S}_{0}(V)=e^{V} \equiv \sum_{n} \frac{V \cdot \ldots \cdot V}{n !}$ ).

Flow operator. From the definition (5.1) it follows

$$
V_{\Lambda}=\mathbf{S}_{\Lambda}^{-1} \circ \mathbf{S}_{\Lambda_{0}}\left(V_{\Lambda_{0}}\right)
$$

i.e. $\mathbf{S}_{\Lambda}^{-1} \circ \mathbf{S}_{\Lambda_{0}}$ is the "flow of the effective potential from $\Lambda_{0}$ to $\Lambda$ ".

We want to clarify the relation between this flow operator and $\mathbf{S}_{\Lambda, \Lambda_{0}}$; by the latter we mean the regularized $S$-matrix with propagator $p_{\Lambda, \Lambda_{0}}$ (4.12), analogously to (4.1) and (4.2). Similarly to (4.6), $\mathbf{S}_{\Lambda, \Lambda_{0}}$ satisfies

$$
\mathbf{S}_{\Lambda, \Lambda_{0}}=\tau_{\Lambda, \Lambda_{0}} \circ \exp \circ \tau_{\Lambda, \Lambda_{0}}^{-1}
$$


where $\tau_{\Lambda, \Lambda_{0}}$ is defined as $\tau_{\Lambda}$ (4.4), with propagator $p_{\Lambda, \Lambda_{0}}$ instead of $p_{\Lambda}$. For $\Lambda=0$ we have $p_{0}=0, p_{0, \Lambda_{0}}=p_{\Lambda_{0}}$, hence $\tau_{0}=\mathrm{id}, S_{0}^{-1}=\log$ and $S_{0, \Lambda_{0}}=S_{\Lambda_{0}}$. With that, $\mathbf{S}_{0}^{-1} \circ \mathbf{S}_{\Lambda_{0}}$ (i.e. the flow from $\Lambda_{0}$ to 0 ) is equal to $\log \circ \mathbf{S}_{0, \Lambda_{0}}$. For the flow from $\Lambda_{0}$ to an arbitrary $\Lambda \in\left[0, \Lambda_{0}\right]$ we assert

$$
\mathbf{S}_{\Lambda}^{-1} \circ \mathbf{S}_{\Lambda_{0}}=\tau_{\Lambda} \circ \log \circ \mathbf{S}_{\Lambda, \Lambda_{0}} \circ \tau_{\Lambda}^{-1}
$$

Proof: We first note that

$$
\tau_{\Lambda, \Lambda_{0}}=\exp \left(i \hbar\left(\Gamma_{\Lambda_{0}}-\Gamma_{\Lambda}\right)\right)=\tau_{\Lambda}^{-1} \circ \tau_{\Lambda_{0}}=\tau_{\Lambda_{0}} \circ \tau_{\Lambda}^{-1}
$$

Due to (4.6) and (5.5) it holds

$$
\mathbf{S}_{\Lambda, \Lambda_{0}}=\tau_{\Lambda, \Lambda_{0}} \circ \exp \circ \tau_{\Lambda, \Lambda_{0}}^{-1}=\tau_{\Lambda}^{-1} \circ \mathbf{S}_{\Lambda_{0}} \circ \tau_{\Lambda}
$$

and with that we obtain

$$
\mathbf{S}_{\Lambda}^{-1} \circ \mathbf{S}_{\Lambda_{0}} \circ \tau_{\Lambda}=\tau_{\Lambda} \circ \log \circ \tau_{\Lambda}^{-1} \circ \mathbf{S}_{\Lambda_{0}} \circ \tau_{\Lambda}=\tau_{\Lambda} \circ \log \circ \mathbf{S}_{\Lambda, \Lambda_{0}}
$$

Flow equation. The flow equation (cf. $[12,14,11,2]$ ) is a differential equation for $V_{\Lambda}$ as a function of $\Lambda$.

\section{Theorem 2.}

$$
\begin{aligned}
\frac{d}{d \Lambda} V_{\Lambda} & = & -\frac{\hbar}{2} \int d x d y \frac{d p_{\Lambda}(x-y)}{d \Lambda} \frac{\delta V_{\Lambda}}{\delta \varphi(x)} \star_{p_{\Lambda}} \frac{\delta V_{\Lambda}}{\delta \varphi(y)} \\
& = & -\left.\frac{1}{2} \frac{d}{d \lambda}\right|_{\lambda=\Lambda}\left(V_{\Lambda} \star_{p_{\lambda}} V_{\Lambda}\right)
\end{aligned}
$$

Proof $^{2}$. From $\mathbf{S}_{\Lambda}(V)=e_{\star_{p_{\Lambda}}}^{V}$ we see that

$$
\frac{d}{d \lambda} \mathbf{S}_{\Lambda}\left(V_{\lambda}\right)=\frac{d V_{\lambda}}{d \lambda} \star_{p_{\Lambda}} \mathbf{S}_{\Lambda}\left(V_{\lambda}\right)
$$

and with that we obtain

$$
0=\frac{d}{d \Lambda} \mathbf{S}_{\Lambda}\left(V_{\Lambda}\right)=\left.\frac{d}{d \lambda}\right|_{\lambda=\Lambda} \mathbf{S}_{\lambda}\left(V_{\Lambda}\right)+\frac{d V_{\Lambda}}{d \Lambda} \star_{p_{\Lambda}} \mathbf{S}_{\Lambda}\left(V_{\Lambda}\right)
$$

Due to $\mathbf{S}_{\Lambda}(F)=1+\mathcal{O}(F)$ the inverse (w.r.t. $\star_{p_{\Lambda}}$ ) regularized $S$-matrix $\mathbf{S}_{\Lambda}(F)^{-1}$ exists. With that it follows that

$$
\frac{d V_{\Lambda}}{d \Lambda}=-\left.\frac{d}{d \lambda}\right|_{\lambda=\Lambda} \mathbf{S}_{\lambda}\left(V_{\Lambda}\right) \star_{p_{\Lambda}} \mathbf{S}_{\Lambda}\left(V_{\Lambda}\right)^{-1}
$$

\footnotetext{
${ }^{2} \mathrm{~A}$ somewhat different proof is given in [2].
} 
From the definition (2.6) of $\star_{p}$ we obtain

$$
\frac{d}{d \Lambda} \frac{F \star_{p_{\Lambda}} F}{2}=\frac{\hbar}{2} \int d x d y \frac{d p_{\Lambda}(x-y)}{d \Lambda} \frac{\delta F}{\delta \varphi(x)} \star_{p_{\Lambda}} \frac{\delta F}{\delta \varphi(y)}
$$

and for $n$ factors

$\frac{d}{d \Lambda} \frac{T_{\Lambda}\left(F^{\otimes n}\right)}{n !}=\frac{\hbar}{2(n-2) !} \int d x d y \frac{d p_{\Lambda}(x-y)}{d \Lambda} \frac{\delta F}{\delta \varphi(x)} \star_{p_{\Lambda}} \frac{\delta F}{\delta \varphi(y)} \star_{p_{\Lambda}} F \star_{p_{\Lambda}} \ldots \star_{p_{\Lambda}} F$

(2 factors $\frac{\delta F}{\delta \varphi}$ and $(n-2)$ factors $F$ ). Summing over $n$ we obtain

$$
\frac{d}{d \Lambda} \mathbf{S}_{\Lambda}(F)=\frac{\hbar}{2} \int d x d y \frac{d p_{\Lambda}(x-y)}{d \Lambda} \frac{\delta F}{\delta \varphi(x)} \star_{p_{\Lambda}} \frac{\delta F}{\delta \varphi(y)} \star_{p_{\Lambda}} \mathbf{S}_{\Lambda}(F)
$$

Inserting this into (5.10) it results (5.8), from which we obtain (5.9) by using (5.11).

Construction of $V_{\Lambda}$. Usually $\mathbf{S}$ is unknown, only $V$ and $\left(p_{\Lambda}\right)_{\Lambda>0}$ are given, and from that $V_{\Lambda}$ is computed by solving the flow equation. In perturbation theory this amounts to an inductive construction of $V_{\Lambda}$ as a formal power series in $V$. Namely, denoting by $V_{\Lambda}^{(n)}$ the term in $V_{\Lambda}$ of order $n$ in $V$, and taking $V_{\Lambda}^{(0)}=0$ into account, the perturbative version of the flow equation reads

$$
\frac{d}{d \Lambda} V_{\Lambda}^{(n)}=\sum_{k=1}^{n-1}-\left.\frac{1}{2} \frac{d}{d \lambda}\right|_{\lambda=\Lambda}\left(V_{\Lambda}^{(k)} \star_{p_{\lambda}} V_{\Lambda}^{(n-k)}\right) .
$$

Proceeding inductively, we start with $V_{\Lambda}^{(1)}=V(5.2)$ and assume that $V_{\Lambda}^{(k)}$ is known for all $k<n$. Then, the r.h.s. is known and, hence, an integration yields $V_{\Lambda}^{(n)}$. A major problem is the determination of the integration constant by a suitable boundary value. (The value (5.3) at $\Lambda=0$ does not help, because it contains the unknown $\mathbf{S}$.) We refer to the usual procedure which is roughly sketched in the next section.

Concerning the removal of the cutoff $\Lambda$, we point out that $V_{\Lambda}$ diverges in general for $\Lambda \rightarrow \infty$. But $\lim _{\Lambda \rightarrow \infty} \mathbf{S}_{\Lambda}\left(V_{\Lambda}\right)$ exists and gives $\mathbf{S}(V)$.

\section{Comparison with the functional integral approach}

First we roughly sketch the usual procedure for the Euklidean theory, following [14]. One defines an effective action $G_{\Lambda, \Lambda_{0}}$ by the functional integral

$$
e^{G_{\Lambda, \Lambda_{0}}(\varphi)}:=\int d \mu_{p_{\Lambda, \Lambda_{0}}}(\phi) e^{-\lambda V^{\left(\Lambda_{0}\right)}(\phi+\varphi)},
$$


where the normalization of the functional integral is included in the Gaussian measure $d \mu_{p_{\Lambda, \Lambda_{0}}}$, the covariance $p_{\Lambda, \Lambda_{0}}$ of $d \mu_{p_{\Lambda, \Lambda_{0}}}$ is given by (4.12), $\lambda$ is the coupling constant and $V^{\left(\Lambda_{0}\right)}$ is the interaction. Heuristically speaking, the 'degrees of freedom in the region $\Lambda^{2} \prec p^{2} \prec \Lambda_{0}^{2}$ are integrated out'. Graphically $G_{\Lambda, \Lambda_{0}}(\varphi)$ is the sum of all connected Feynman diagrams with vertices $\lambda V^{\left(\Lambda_{0}\right)}$, internal lines symbolizing $p_{\Lambda, \Lambda_{0}}$ and external lines symbolizing the field $\varphi$.

The interaction $V^{\left(\Lambda_{0}\right)}$ is usually local and depends on $\Lambda_{0}$ since it is normally ordered with respect to $p_{0, \Lambda_{0}}$ (or $p_{\Lambda, \Lambda_{0}}$ ) and because it contains $\Lambda_{0^{-}}$ dependent local counterterms as explained in (6.2), (6.3) below.

A main difference to our formalism is that there the interaction $V$ has compact support (see (2.1)); but this does not hold here, e.g. for the $\varphi^{n}$-model the unrenormalized interaction (i.e. without counterterms) reads

$$
\int d^{d} x \Omega_{p}\left((\varphi(x))^{n}\right), \quad \text { where } \Omega_{p}(\ldots) \text { denotes normal ordering w.r.t. } p \text {. }
$$

Therefore, in our formalism IR-divergences do not occur; but here they can appear and, hence, in general it is necessary to introduce the IR-cutoff $\Lambda>0$. Purely massive models are an exception: they are IR-finite also in the usual formalism and, hence, one can set $\Lambda=0$.

Computing $\frac{\partial}{\partial \Lambda}$ of the functional integral (6.1) one derives the flow equation. Let $G_{\Lambda, \Lambda_{0}}^{(r)}$ be that term of $G_{\Lambda, \Lambda_{0}}$ which is of order $r$ in the coupling constant $\lambda$ (or equivalently in $V$ ). Proceeding by induction on $r$, the flow equation expresses $\frac{\partial G_{\Lambda, \Lambda_{0}}^{(r)}}{\partial \Lambda}$ in terms of lower order terms $G_{\Lambda, \Lambda_{0}}^{(k)}, k<r$, which are inductively known. Solving the flow equation

$$
G_{\Lambda, \Lambda_{0}}^{(r)}=G_{\Lambda_{0}, \Lambda_{0}}^{(r)}-\int_{\Lambda}^{\Lambda_{0}} d \Lambda^{\prime} \frac{\partial G_{\Lambda^{\prime}, \Lambda_{0}}^{(r)}}{\partial \Lambda^{\prime}}
$$

(where $\frac{\partial G_{\Lambda^{\prime}, \Lambda_{0}}^{(r)}}{\partial \Lambda^{\prime}}$ is expressed in terms of inductively known terms by the flow equation) there appears the crucial question how to choose the boundary value $G_{\Lambda_{0}, \Lambda_{0}}$. Choosing for $G_{\Lambda_{0}, \Lambda_{0}}$ the unrenormalized (normally ordered) interaction $-\lambda \Omega_{p}(V)$, the limit $\lim _{\Lambda_{0} \rightarrow \infty} G_{\Lambda, \Lambda_{0}}$ does not exist in general (due to the usual UV-divergences). Therefore, one adds $\Lambda_{0}$-dependent local counterterms,

$$
G_{\Lambda_{0}, \Lambda_{0}}=-\lambda V^{\left(\Lambda_{0}\right)}=-\lambda \Omega_{p}(V)+\Lambda_{0} \text {-dependent local counterterms },
$$


such that this limit exists. The theory is 'perturbatively renormalizable' if this is possible by a finite number of counterterms (each counterterm may be a formal power series in $\lambda$ ). In case of the $\phi^{4}$-interaction in $d=4$ dimensions one has to add three counterterms of the form

$G_{\Lambda_{0}, \Lambda_{0}}=\lambda \Omega_{p}\left(\phi^{4}\right)\left(-1+\sum_{r \geq 1} c_{\Lambda_{0}, r} \lambda^{r}\right)+\Omega_{p}\left(\phi^{2}\right) \sum_{r \geq 2} a_{\Lambda_{0}, r} \lambda^{r}+\Omega_{p}\left((\partial \phi)^{2}\right) \sum_{r \geq 2} b_{\Lambda_{0}, r} \lambda^{r}$,

where $a_{\Lambda_{0}, r}, b_{\Lambda_{0}, r}, c_{\Lambda_{0}, r}$ are $\Lambda_{0^{-}}$dependent numbers.

We now compare with our formalism.

- $\tau_{\Lambda, \Lambda_{0}}$ and $\mathbf{S}_{\Lambda, \Lambda_{0}}$ as functional integrals: for $F \in \mathcal{F}$

$$
\left(\tau_{\Lambda, \Lambda_{0}} F\right)(\varphi) \text { corresponds to } \int d \mu_{p_{\Lambda, \Lambda_{0}}}(\phi) F(\phi+\varphi)
$$

since both expressions are the sum over all possible contractions of $\varphi$ in $F(\varphi)$ with propagator $p_{\Lambda, \Lambda_{0}}$.

Moreover let $\mathbf{S}_{\Lambda, \Lambda_{0}}$ be the regularized $S$-matrix with propagator $p_{\Lambda, \Lambda_{0}}$. Then, for $V \in \mathcal{F}$,

$$
S_{\Lambda, \Lambda_{0}}(\lambda V)(\varphi) \text { corresponds to } \int d \mu_{p_{\Lambda, \Lambda_{0}}}(\phi) e^{-\lambda \Omega_{p_{\Lambda, \Lambda_{0}}}(V(\phi+\varphi))}
$$

since for both expressions the term $\sim \lambda^{n}$ is the sum over all contractions (with propagator $p_{\Lambda, \Lambda_{0}}$ ) between $n$ vertices, each vertex given by $V$. Note that in the functional integral selfcontractions of a vertex (i.e. tadpoles) drop out due to the normal ordering of $V$ w.r.t. $p_{\Lambda, \Lambda_{0}}$.

Even for $F, V \in \mathcal{F}_{\text {loc }}$ all expressions in (6.4) and (6.5) are well-defined (i.e. renormalization is not needed at this level) since $p_{\Lambda, \Lambda_{0}} \in \mathcal{S}\left(\mathbb{R}^{d}\right)$.

- Effective potential: Our effective potential

$$
V_{\Lambda}:=\mathbf{S}_{\Lambda}^{-1} \circ \mathbf{S}(V) \text { corresponds roughly to } G_{\Lambda, \infty}:=\lim _{\Lambda_{0} \rightarrow \infty} G_{\Lambda, \Lambda_{0}}
$$

For $\Lambda=0$ these expressions agree: namely in our formalism we have the value

$$
e^{V_{0}(\varphi)}=\mathbf{S}(V)(\varphi)
$$


(see (5.3)), which is a main justification to interprete $V_{\Lambda}$ as effective potential. On the other side, in an IR-finite model, the functional integral

$$
\lim _{\Lambda_{0} \rightarrow \infty} e^{G_{0, \Lambda_{0}}(\varphi)}=\lim _{\Lambda_{0} \rightarrow \infty} \int d \mu_{p_{0, \Lambda_{0}}}(\phi) e^{-\lambda V^{\left(\Lambda_{0}\right)}(\phi+\varphi)}
$$

gives also $\mathbf{S}(V)(\varphi)$.

As mentioned above, the existence of $\lim _{\Lambda_{0} \rightarrow \infty} G_{\Lambda, \Lambda_{0}}$ involves renormalization that is the addition of suitable local counterterms. Also the definition (1.2) of $V_{\Lambda}$ presupposes renormalization, since $V_{\Lambda}$ is defined in terms of the renormalized $S$-matrix.

- UV-finite models: in an UV-finite theory (e.g. interaction $\phi^{n}$ in $d=2$ dimensions or $\phi^{2}$ for $\left.d=3\right) V^{\left(\Lambda_{0}\right)}(6.2)$ depends on $\Lambda_{0}$ only by normal ordering. If the latter is done with respect to $p_{\Lambda, \Lambda_{0}}$, we see from (6.1) and (6.5) that

$$
S_{\Lambda, \Lambda_{0}}(\lambda V)(\varphi) \text { corresponds to } e^{G_{\Lambda, \Lambda_{0}}(\varphi)}
$$

Taking also

$$
\mathbf{S}(F)=\lim _{\Lambda_{0} \rightarrow \infty} \mathbf{S}_{\Lambda_{0}}(F), \quad \forall F \in \mathcal{F}_{\text {loc }},
$$

(see (6.14) below) and (5.7) into account and choosing $\lambda=1$ we obtain

$$
\begin{aligned}
G_{\Lambda, \infty} & \simeq \lim _{\Lambda_{0} \rightarrow \infty} \log \circ \mathbf{S}_{\Lambda, \Lambda_{0}}(V) \\
& =\lim _{\Lambda_{0} \rightarrow \infty} \log \circ \tau_{\Lambda}^{-1} \circ \mathbf{S}_{\Lambda_{0}} \circ \tau_{\Lambda}(V)=\log \circ \tau_{\Lambda}^{-1} \circ \mathbf{S} \circ \tau_{\Lambda}(V) .
\end{aligned}
$$

Usually this differs from

$$
V_{\Lambda}=\mathbf{S}_{\Lambda}^{-1} \circ \mathbf{S}(V)=\tau_{\Lambda} \circ \log \circ \tau_{\Lambda}^{-1} \circ \mathbf{S}(V)
$$

an exception is $\Lambda=0$ (as generally noted in (6.7), (6.8)). Or, arguing somewhat differently: the difference between $V_{\Lambda}(6.12)$ and $G_{\Lambda, \infty}(6.11)$ amounts to the difference between $\mathbf{S}_{\Lambda}^{-1} \circ \mathbf{S}_{\Lambda_{0}}$ and $\log \circ \mathbf{S}_{\Lambda, \Lambda_{0}}$ for $\Lambda_{0} \rightarrow \infty$, which is given by (5.6). The maps $V \mapsto V_{\Lambda}$ (6.12) and $V \mapsto G_{\Lambda, \infty}$ (6.11) agree up to a similarity transformation by $\tau_{\Lambda}$, which is a matter of convention. 
Relation of Wilson's RG to the Stückelberg-Petermann RG (heuristic treatment). The difference in the conventions for $V_{\Lambda}$ and $G_{\Lambda, \infty}((6.12)$ versus (6.11)) is no obstacle to interprete $V_{\Lambda}$ as an effective potential and, hence, to interprete the corresponding flow operators

$$
\left\{\mathbf{S}_{\Lambda}^{-1} \circ \mathbf{S}_{\Lambda_{0}} \mid 0 \leq \Lambda \leq \Lambda_{0}<\infty\right\}
$$

as a version of Wilson's $R G$. Proceeding heuristically, we are now going to show that the restriction of the operators (6.13) to $\mathcal{F}_{\text {loc }}$ can be approximated by a subfamily of the Stückelberg-Petermann RG, for $\Lambda, \Lambda_{0}$ big enough.

For this purpose we use that, for a renormalizable model, the limit $\Lambda \rightarrow \infty$ of $\mathbf{S}_{\Lambda}$ exists if one adds suitable local counterterms (analogously to (6.2), (6.3)). This addition of local counterterms can be described by $V \rightarrow Z_{\Lambda}(V)$ with an element $Z_{\Lambda}$ of the Stückelberg-Petermann group. In detail [2]: for all $0<\Lambda<\infty$ there exists a $Z_{\Lambda} \in \mathcal{R}_{0}$ with

$$
\lim _{\Lambda \rightarrow \infty} \mathbf{S}_{\Lambda} \circ Z_{\Lambda}=\mathbf{S}
$$

By $\mathcal{R}_{0}$ we mean the version of the Stückelberg-Petermann RG which is defined by requiring only the conditions Starting element, Locality and Field Independence. Since usually $\mathbf{S}_{\Lambda}$ does not satisfy Poincaré invariance, almost homogeneous Scaling and Unitarity (and possibly violates also Smoothness in $m \geq 0$ ), we may not expect that $Z_{\Lambda}$ fulfils the corresponding conditions.

With (6.14) we have $\lim _{\Lambda \rightarrow \infty} \mathbf{S}_{\Lambda} \circ Z_{\Lambda}=\mathbf{S}=\lim _{\Lambda_{0} \rightarrow \infty} \mathbf{S}_{\Lambda_{0}} \circ Z_{\Lambda_{0}}$, from which we conclude that

$$
\left.\mathbf{S}_{\Lambda}^{-1} \circ \mathbf{S}_{\Lambda_{0}}\right|_{\mathcal{F}_{\text {loc }}} \approx Z_{\Lambda} \circ Z_{\Lambda_{0}}^{-1} \in \mathcal{R}_{0} \quad \text { for } \quad \Lambda, \Lambda_{0} \rightarrow \infty
$$

That is, for $\Lambda, \Lambda_{0}$ big enough, the restriction of the flow operators $\mathbf{S}_{\Lambda}^{-1} \circ \mathbf{S}_{\Lambda_{0}}$ to $\mathcal{F}_{\text {loc }}$ can be approximated by the 2-parametric subfamily $Z_{\Lambda} \circ Z_{\Lambda_{0}}^{-1}$ of the Stückelberg-Petermann group $\mathcal{R}_{0}$.

Acknowledgments. The author was supported by the Deutsche Forschungsgemeinschaft through the Institutional Strategy of the University of Göttingen.

This article is based on reference [2] - a joint work with Romeo Brunetti and Klaus Fredenhagen. Many discussions with Romeo and Klaus have been very helpful. 


\section{References}

[1] Brennecke, F., Dütsch, M., "Removal of violations of the Master Ward Identity in perturbative QFT," Rev. Math. Phys. 20 (2008), 119-172

[2] Brunetti, R., Dütsch, M., Fredenhagen, K., "Perturbative Algebraic Quantum Field Theory and the Renormalization Groups", Adv. Theor. Math. Phys. 13 (2009) 1541-1599

[3] Brunetti, R., Fredenhagen, K., "Microlocal analysis and interacting quantum field theories: Renormalization on physical backgrounds", Commun. Math. Phys. 208 (2000) 623

[4] Dütsch, M., Fredenhagen, K., "Algebraic Quantum Field Theory, Perturbation Theory, and the Loop Expansion," Commun. Math. Phys. 219 (2001) 5

[5] Dütsch, M., Fredenhagen, K., "Perturbative algebraic field theory, and deformation quantization," Fields Institute Communications 30 (2001), $151-160$

[6] Dütsch, M., Fredenhagen, K., "Causal perturbation theory in terms of retarded products, and a proof of the Action Ward Identity," Rev. Math. Phys. 16 (2004) 1291-1348

[7] Dütsch, M., Fredenhagen, K., "Action Ward Identity and the Stückelberg-Petermann renormalization group," in 'Rigorous Quantum Field Theory', editors A. Boutet de Monvel, D. Buchholz, D. Iagolnitzer, U. Moschella, Birkhäuser Verlag (2006) 113-123

[8] Epstein, H., Glaser, V., "The role of locality in perturbation theory," Ann. Inst. H. Poincaré A 19 (1973) 211

[9] Hollands, S., Wald, R. M., "On the Renormalization Group in Curved Spacetime," Commun. Math. Phys. 237 (2003) 123-160

[10] Hörmander, L., The analysis of linear partial differential operators. I. Distribution theory and Fourier analysis. Springer Study Edition. Springer-Verlag, Berlin, (1990) 2nd ed. 
[11] Keller, G., Kopper, C., Schophaus, C., "Perturbative renormalization with flow equations in Minkowski space," Helv. Phys. Acta 70 (1997) $247-274$

[12] Polchinski, J., "Renormalization and Effective Lagrangians," Nucl. Phys. B231 (1984) 269-295

[13] Popineau, G., Stora, R., "A pedagogical remark on the main theorem of perturbative renormalization theory", unpublished preprint (1982)

[14] Salmhofer, M., Renormalization. An introduction. Texts and Monographs in Physics. Springer-Verlag, Berlin, (1999)

[15] Stora, R., "Differential algebras in Lagrangean field theory", ETHZürich Lectures, January-February 1993

[16] Stückelberg, E.C.G., Petermann, A., "La normalisation des constantes dans la théorie des quanta," Helv. Phys. Acta 26 (1953) 499-520 